\title{
Barriers Detection Method for Stereovision-Based ACC Systems
}

\author{
Tiberiu Marița \\ Technical University of Cluj-Napoca \\ E-mail:Tiberiu.Marita@cs.utcluj.ro
}

\begin{abstract}
This paper presents a vision-based road-barriers detection method. Because horizontal structures are hard to detect by binocular stereovision, object detection methods based on 3D points grouping fail to detect the barriers as obstacles and consequently specific ACC applications as longitudinal control will fail to react when the vehicle path is obstructed by such an object. Therefore the proposed method combines the detection of the horizontal structure of a barrier's boom in the 2D grayscale image space using a Hough based approach followed by a series of $3 D$ validation steps based on dense stereo-vision and derived functions (lane and objects detection) in order to eliminate false positives and to infer the associated $3 D$ information. The detected barrier objects are reported as $3 D$ cuboids which are further tracked along with other objects/obstacles detected through a $3 D$ points grouping method integrated in a multifunction application for vision based driving assistance.
\end{abstract}

\section{Introduction}

One of the main functions of an ACC (Active Cruise Control) system with Stop\&Go function used in vehicles [1-3] is the longitudinal control. Its aim is to maintain a safety clearance relative to the preceding vehicles or to stop the vehicle if an obstacle obstructs the vehicle's path. Most of the commercially available ACC systems are based on well established and relatively chip radar sensors technology but are usable only in limited scenarios (straight roads and a minimal speed limit) [4-5]. Urban ACC systems have to face more complex situations (difficult and atypical road geometries, crowded traffic, presence of pedestrians and other type of traffic participants which are not normally found on the highways or country roads). In such situations the use of vision sensors is compulsory.
Dense-stereo vision sensors [6],[7] have been proved to be the most reliable ones in such scenarios and are able to detect and track clothoid and nonclothoid lanes, drivable areas in the absence of lane markings, cars, pedestrians and other types of obstacles [8].

Due to the configuration of the binocular stereo vision systems (horizontal displacement of the cameras), horizontal features are hard to reconstruct (due to matching ambiguities [9]). Therefore, objects containing only horizontal features/edges have sparse and mainly erroneous 3D information associated and cannot be detected in the classical way (by grouping of 3D points [9],[10]). This is one of the reasons while this topic of research is missing completely from the literature.

The proposed solution for detecting horizontal structures is to combine the detection in the $2 \mathrm{D}$ image followed by $3 \mathrm{D}$ validation/classification. The 2D detection is based on a Hough based approach which is used to detect only horizontal lines from the edge map of a grayscale image. The detected horizontal lines are further used as candidates for barriers' detection. These horizontal structures can belong to different classes of objects, as barriers (horizontal structures placed above the road), road features (transversal road markings or curbs etc), or other unclassifiable horizontal structures. Therefore, a series of validations steps based on primary dense 3D data and higher level processing outputs as road surface parameters provided by a lane detection module [11] and 3D obstacles provided by an object detection module [10]) are performed in order to eliminate the false positives. The detected barrier objects are reported as 3D cuboids which are further tracked along with other objects/obstacles detected through a $3 \mathrm{D}$ points grouping method integrated in a multi-function application for vision based driving assistance.

The system was tested to detect various barriers-like structures as toll check-points, parking entrances, railway cross points etc. providing real time detection capabilities. 


\section{Problem statement}

Most of the barriers' booms exhibit few 3D reconstructed points even using a dense stereo vision engine (with the two cameras displaced horizontally). This can be explained by the impossibility to correlate long horizontal edge segments which appear at the boom-background frontier (Figure 1.a). Even if the boom's surface has some artifacts which can be correlated (Figure 1.b), the 3D points are so sparse that are ignored by a grouping algorithm [8] which groups $3 \mathrm{D}$ points in objects on density and vicinity criteria. The same applies to other horizontal structures as transversal road markings (Figure 1.c)

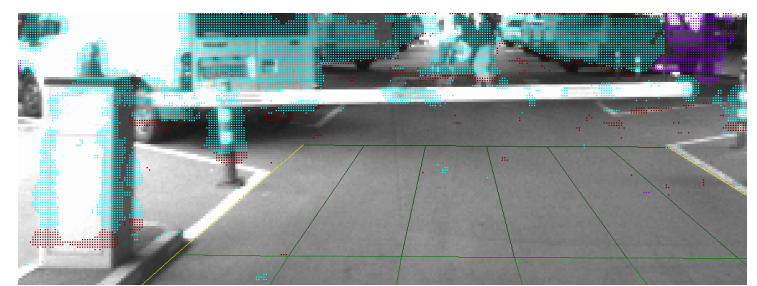

a. Barrier with no painted artifacts: few points are reconstructed.

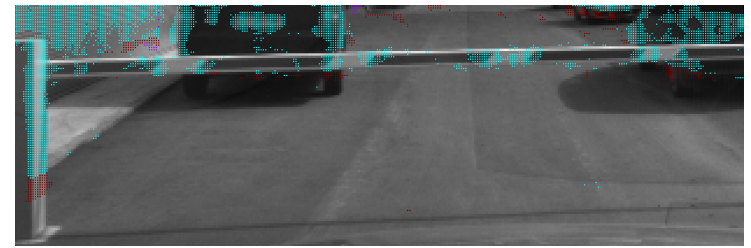

b. Barrier with painted artifacts: only regions around their vertical edges are reconstructed.

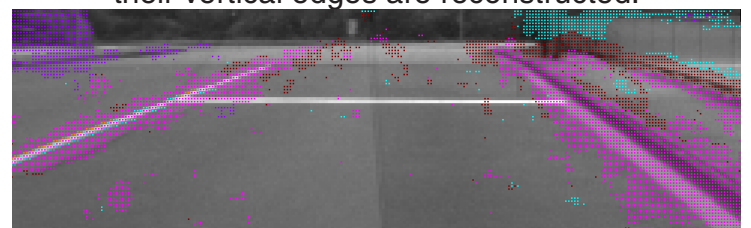

c. Transversal road marking: only points form its extremities are reconstructed.

Figure 1. Reconstruction of different types of horizontal structures. 3D points above the road surface are figured with cyan, while road points with magenta.

The proposed solution uses a hybrid approach. First it detects the horizontal structures in 2D (using horizontal edges and lines detection through the Hough transform). The horizontal line segments detected in 2D will be used as search regions for 3D points. Even if these 3D points are very sparse, they can offer enough information for discriminating barrier like structures. The main steps of the barriers detection algorithm along with supplementary validation criteria are described in the next chapter

\section{The barriers' detection algorithm}

The flow-chart of the proposed barriers detection algorithm is sketched in Figure 8 and detailed bellow:

The first step (1) is to limit the search region to a 3D-ROI (Region Of Interest) in the front of the ego car in which a barrier could be found and detected. Experimental values used are: Width $\approx 2.0 \sim 3.5 \mathrm{~m}$, Height $\approx 0.7 \sim 1.3 \mathrm{~m}$, Depth $\approx 1.0 \sim 15 \mathrm{~m}$. The values are configurable and the limits depend on the stereo system's parameters (lens focal length, horizontal field of view, image resolution, baseline length, maximum disparity). The 3D-ROI is projected on the left image to obtain a 2D-ROI used for searching the horizontal structures in the image (Figure 2).

The second step (2) consists in a trivial horizontal edges detection method [12]. It will detect mainly the edges from the barriers' boom horizontal frontiers (Figure 2).

The third step (3) consists in the detection of horizontal line segments (HLS) using the Hough transform [13]. The Hough space is limited to angles around the horizontal (90 deg.) and points inside the 2D-ROI. The output of the Hough transform is a set of quasi-horizontal line segments having the following parametric equation:

$$
\rho=x \cdot \cos (\theta)+y \cdot \sin (\theta)
$$

where: $\rho$ is the distance from the image coordinate system's origin to the line segment and $\theta$ is the angle between the current line segment and the horizontal (x) image axis (Figure 2).

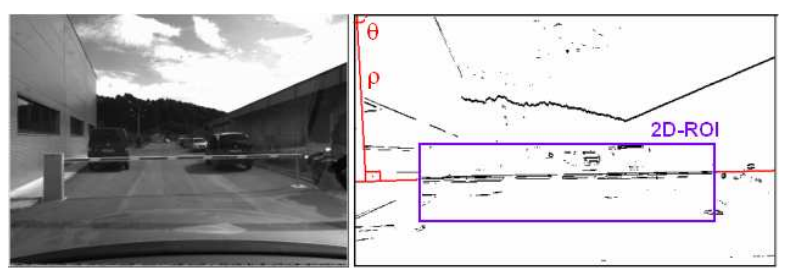

Figure 2. Left: grayscale image with barrier; Right: illustration of the 2D-ROI and Hough lines parameters on the horizontal edge image.

Depending on the number of edge points and Hough transform settings (angular resolution, $\Delta \theta$, and radial resolution, $\Delta \rho$ ) it is possible to have more line segments for one horizontal structure. Therefore an optional step (3.2) can be applied to group HLS upon their 2D vicinity: two or more lines are merged together if $\Delta \rho<2$ [pixels] and $\Delta \theta<2$ [deg]. Performing this step can reduce the processing time by providing less HLS but with the drawback of reducing the accuracy of detected 3D coordinates of the HLS. 
Forth step (4) is the classification of the detected Hough lines in the 2D images using associated 3D information (if available). The following 3 classes can be inferred from the 3D information:

- Barriers line segments (BLS): horizontal line segments placed above the road: $7 \ldots 1.3 \mathrm{~m}$ height (Figure 3.a).

- Road line segments (RLS): horizontal line segments placed on the road (height $\approx 0 \mathrm{~m}$ ) corresponding to transversal markings or curbs (Figure 3.b)

- Unknown line segments (ULS): not belonging to any of the first two classes.
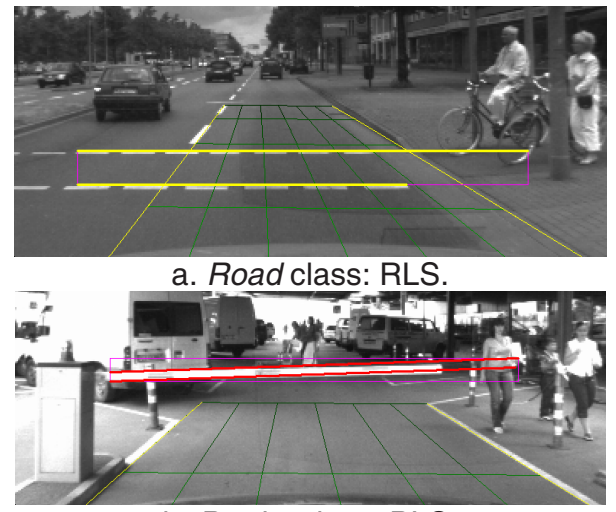

b. Barrier class: BLS

Figure 3. 3D classification of the HLS.

The 3D classification procedure has the following flow:

- The 2D ROI is reduced dynamically (search space reduction) to a rectangle bounding the detected Hough line segments (Figure 3 - magenta color).

-For every 3D point $i$ inside a predefined 3D search tunnel (Figure 5.b), the algorithm checks if its 2D image projection is in the neighborhood of a horizontal line segment ("belongs" to it):

$$
d=\left|y_{i}-\frac{\left(\rho-x_{i} \cdot \cos (\theta)\right)}{\sin (\theta)}\right|<\varepsilon
$$

where: $\left(x_{\mathrm{i}}, y_{\mathrm{i}}\right)$ are the image coordinates of the 3D point $i, \rho$ and $\theta$ are the Hough line (HLS) parameters, $\mathrm{d}$ is the distance from the $3 \mathrm{D}$ point to the HLS (Figure 4).

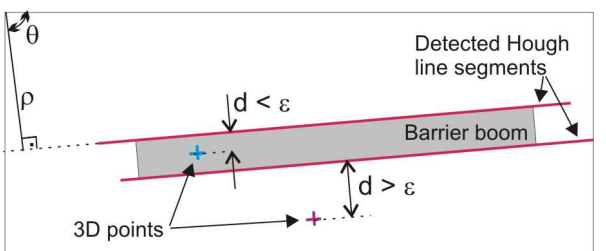

Figure 4. Association of the 3D points with the detected Hough segments (HLS).
-If the 3D point $i$ "belongs" to a horizontal line segment the "class-counters" of the two classes (barrier - BLS or road - RLS) are incremented depending on its 3D coordinates.

- Finally, if the class-counters are above a specific threshold the line segments are given to the specific class.

- For every classified HLS, its average height ( $\left.\mathrm{Y}_{\mathrm{AVG}}\right)$ and depth $\left(\mathrm{Z}_{\mathrm{AVG}}\right)$ are computed (through their associated 3D points). The height is computed relative to the road surface at the computed depth. As a supplementary validation, the standard deviation $\left(\sigma_{\mathrm{Z}}\right)$ of the depths of the containing $3 \mathrm{D}$ points is computed. If $\sigma_{Z}$ is bigger than a specified ratio from $\mathrm{Z}_{\mathrm{AVG}}$, the line segment is rejected (due to erroneous 3D points or different overlapped horizontal structures which are not belonging to a single object).
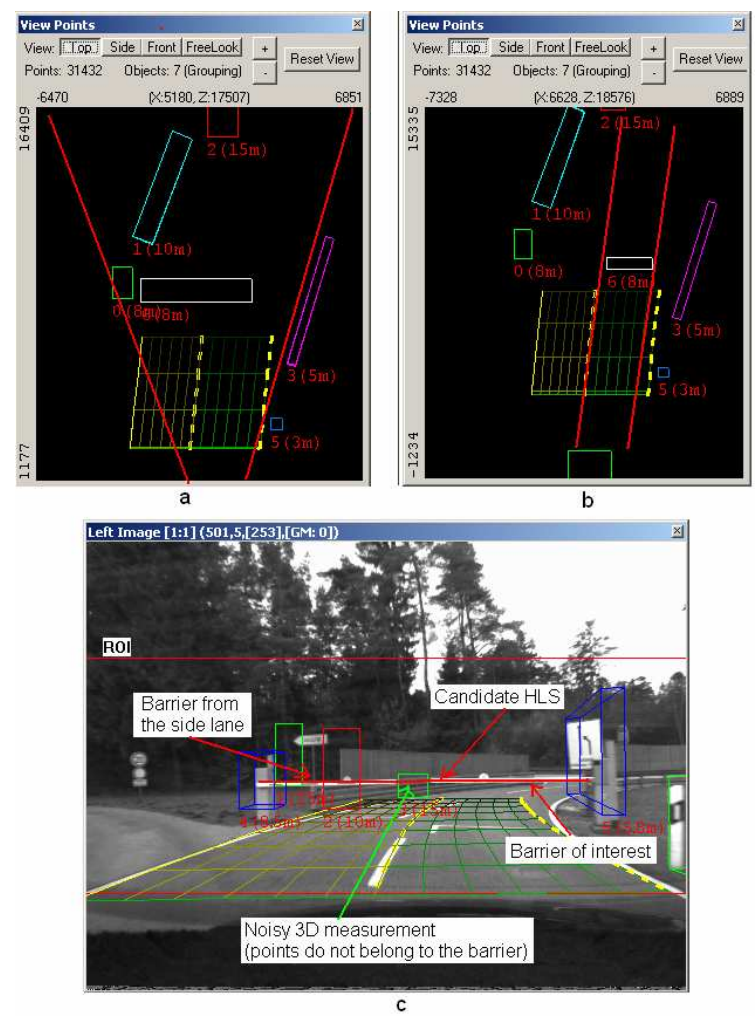

Figure 5. a. Top view of the 3D search space given by the stereo HFOV; b. Top view of the 3D search tunnel following the current lane - green grid delimited with yellow dotted lines (detected barrier colored white);

c. Noisy 3D points not belonging to the barrier of interest are not taken into account.

The 3D search tunnel was introduced to reduce the false positives rate and to increase the measurement accuracy. It has a rectangular shape in the $\mathrm{XOZ}$ 
(transversal) section of the ego-car coordinate system (Figure 5) and follows the 3D surface of the current lane (if exists - Figure 5.b) or has the direction of the longitudinal axis of the car (if lane does not exits). This approach prevents from considering in the current classification step all 3D points inside the horizontal field of view (HFOV) of the stereo system (Figure 5.a), which might not belong to the barrier of interest (Figure 5.c).

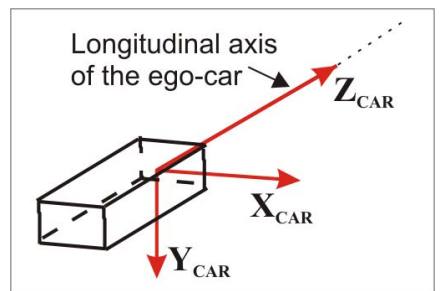

Figure 6. The ego-car coordinate system.

It could be possible to have more then one BLS detected for a barrier (Figure 3.b). The chosen solution (5-th step) was to group them in the YOZ space (Figure $6)$. Having the average height $\left(\mathrm{Y}_{\mathrm{AVG}}\right)$ and depth $\left(\mathrm{Z}_{\mathrm{AVG}}\right)$ for each detected horizontal structures, a clustering process (Modified Basic Sequential Algorithm Scheme - MBSAS [14]) is applied in the 3D space in order to group the BLS upon vicinity criteria in the (YOZ) plane. The average height $\left(\mathrm{Y}_{\mathrm{AVG}}\right)$ and depth $\left(\mathrm{Z}_{\mathrm{AVG}}\right)$ of each cluster (centroid of the class) is computed from the values of its members (containing BLS - figure 7).

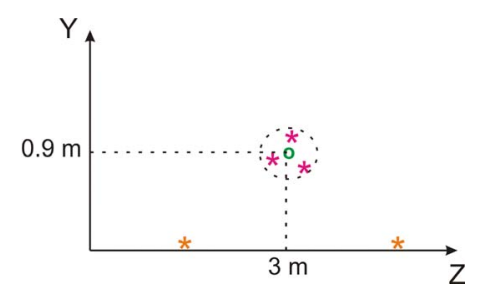

Figure 7. Illustration of the BLS clustering in the YOZ plane by vicinity criteria.

For each BLS-cluster a horizontal, un-oriented, 3D object structure is created (6-th sep). The size and limits of the barrier objects have to fulfill some constraints in order to be trackable (by the objects tracking module [15]): to have a back-face size similar to the one of a car ( $\mathrm{w} \times \mathrm{h}=2 \times 1.5 \mathrm{~m}$ ) and to be a nonfloating objects. Therefore the following dimensions were considered: width $\approx 2 \mathrm{~m},\left|\mathrm{Y}_{\min }\right| \approx 0 \mathrm{~m},\left|\mathrm{Y}_{\max }\right| \approx 1.3$ $\mathrm{m}$

Finally (7-th step), the barrier objects are inserted in the coarse objects list (detected by the grouping module [10]), if there is no overlapping (in the $\mathrm{XOZ}$ view) with an existing object. The objects from the coarse objects list are further feed to the tracking module [15] (Figure 11).

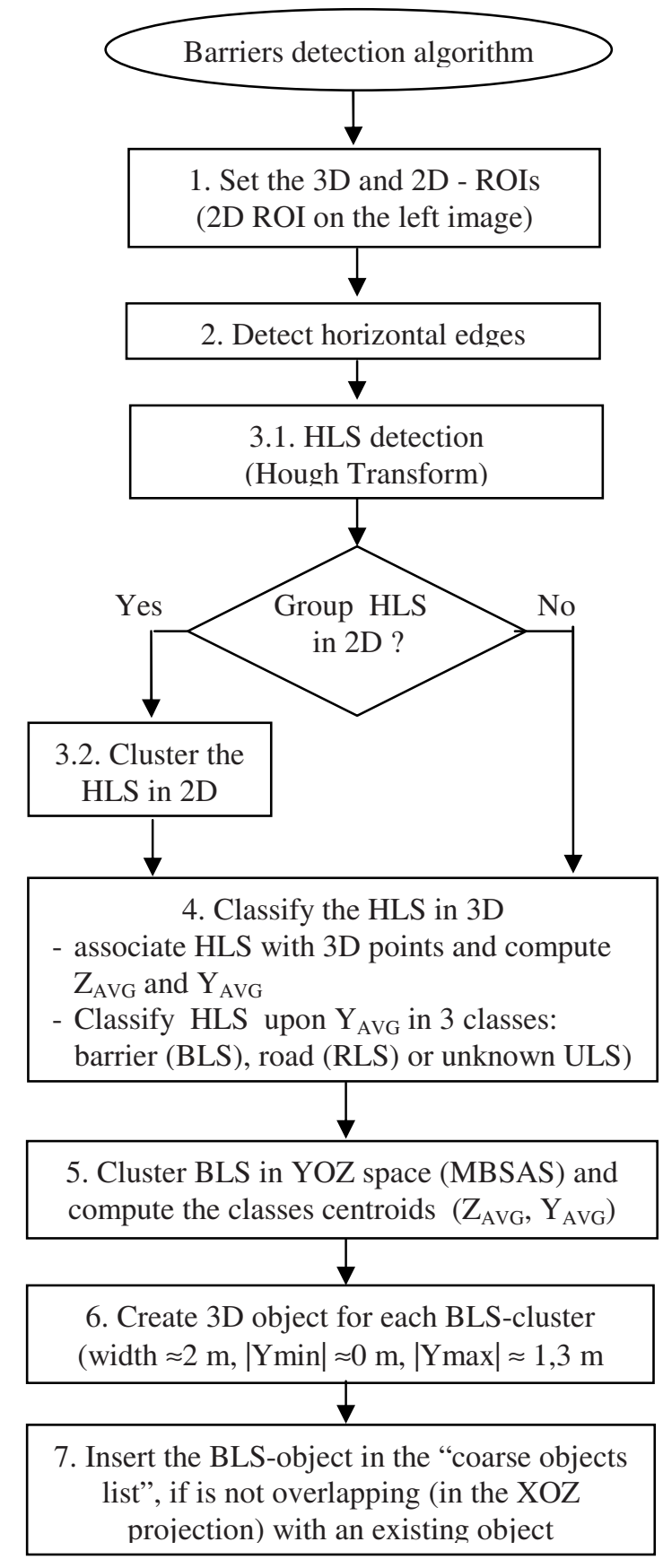

Figure 8. Flow chart of the barriers' detection algorithm.

The non-overlapping condition from the last step was introduced to cope with situations where $3 \mathrm{D}$ points assigned to the detected HLS do not belong to a barrier but to some 3D coarse objects (Figure 9). As a 
consequence these candidate HLS are generating false barrier objects (Figure 9.b).
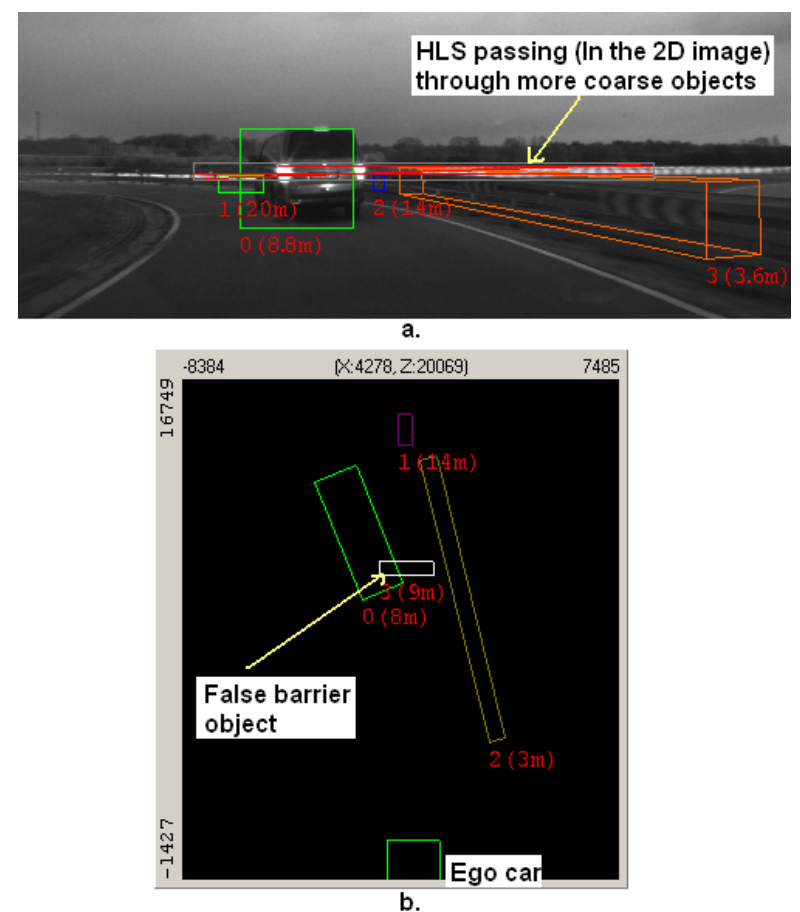

Figure 9. False positive situation: a. Candidate HLS are passing in the 2D through the image projection of some coarse 3D objects; b. False barrier objects are added into the coarse objects list (top view - XOZ).

The solution proposed to solve this problem was to restrict the insertion of the candidate barrier objects into the coarse objects list if the barrier object overlaps (in a certain top-view area percentage) an existing coarse object (Figure 10).
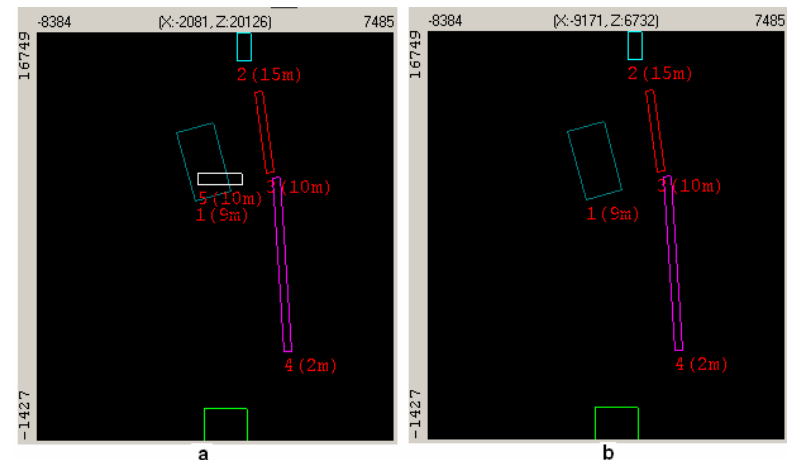

Figure 10. Overlapping rejection of a candidate barrier: $100 \%$ (a) or $10 \%$ (b) are the overlapping ratios allowed.

If more coarse barrier objects are detected at consecutive near depths (due to $3 \mathrm{D}$ reconstruction errors or noisy $3 \mathrm{D}$ points or very relaxed barrier lines clustering conditions), only the nearest coarse barrier object is retained.

\section{The integration of the barrier detection module}

The integration of the barrier detection module in a stereo-vision based application for driving assistance systems [8] is presented in the flowchart from Figure 11. The input dependences of the barriers' detection module are the list of $3 \mathrm{D}$ reconstructed points, the vertical profile of the road (if detected by the lane detection module) and the horizontal profile of the current lane's center (if available). The output of the barrier detection module is a list of detected barrier objects which are appended to the coarse objects' list. The updated coarse object's list is further feed to the tracking module.

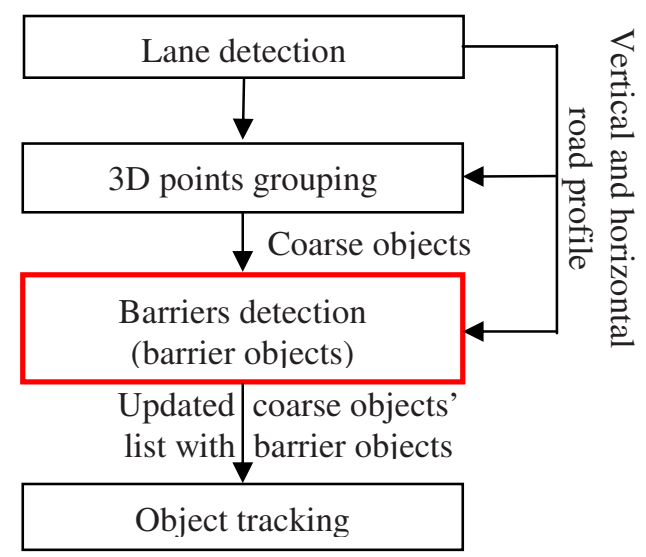

Figure 11. Integration of the barrier detection module.

\section{Results}

Accuracy of the average depth and height of the detected barrier objects was assessed by comparison with the average coordinates of the individual 3D points from the barrier's surface (Figure 12).

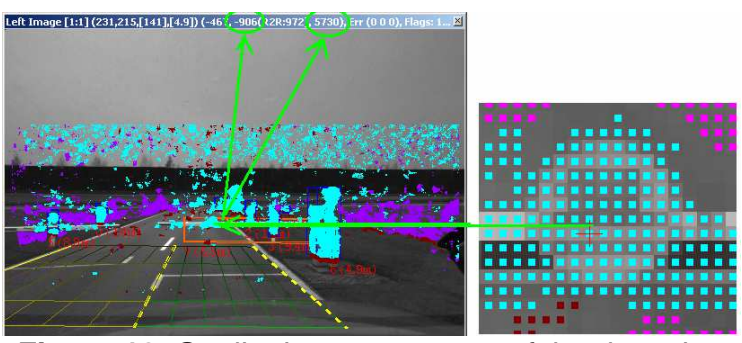

Figure 12. Qualitative assessment of the detection accuracy. 
For example, in the scenario presented in Figure 5, the ground truth established from the $3 \mathrm{D}$ coordinates of the contained points was $8 \mathrm{~m}$ for the depth. The measured depths of the detected barriers using the two types of 3D search regions are given in the table bellow:

Table 1. Depth estimation

\begin{tabular}{|l|l|l|}
\hline $\begin{array}{l}\text { Ground } \\
\text { truth } \\
\text { from 3D } \\
\text { points }\end{array}$ & $\begin{array}{l}\text { Barrier detected } \\
\text { using a 3D-ROI } \\
\text { limited by the } \\
\text { stereo HFOV - } \\
\text { Figure 5.a) }\end{array}$ & $\begin{array}{l}\text { Barrier detected using } \\
\text { a rectangular 3D-ROI } \\
\text { following the current } \\
\text { lane's surface - } \\
\text { Figure 5.b) }\end{array}$ \\
\hline \multicolumn{1}{|c|}{$8.9 \mathrm{~m}$} & \multicolumn{1}{|c|}{$8.1 \mathrm{~m}$} \\
\hline
\end{tabular}

Detection range depends mainly on the stereo camera configuration. For a setup able to perform dense $3 \mathrm{D}$ reconstruction in a range between 0.7 and 35 $\mathrm{m}$ (in front of the ego-car), the barriers detection range established experimentally covers the $0.7 \mathrm{~m} \ldots 12 \mathrm{~m}$ limits, for a barrier with enough artifacts (vertical edges) and good lighting conditions (Figure 13 a,b).

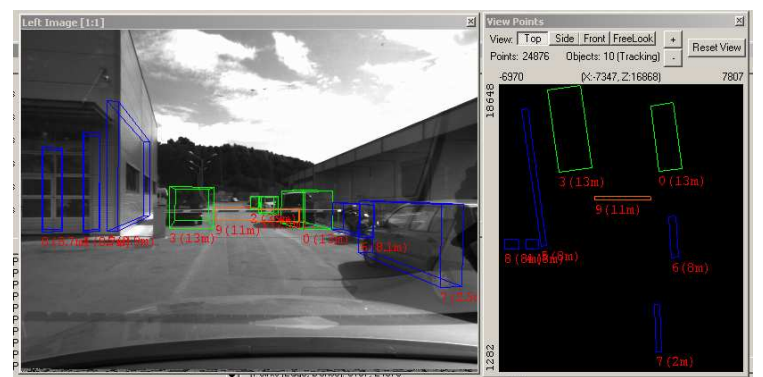

a. Detected barrier at $11.8 \mathrm{~m}$.

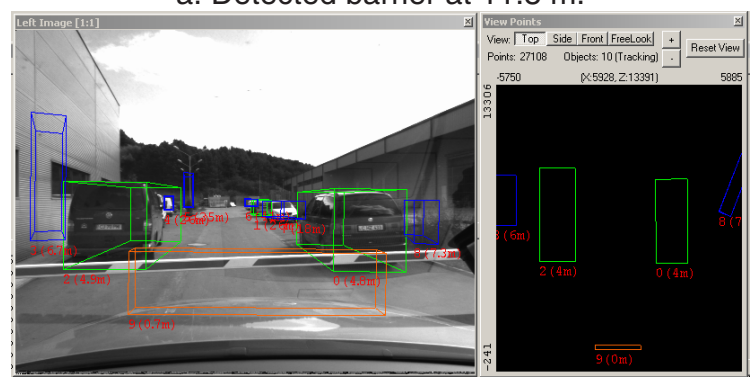

b. Same barrier detected at $0.7 \mathrm{~m}$.

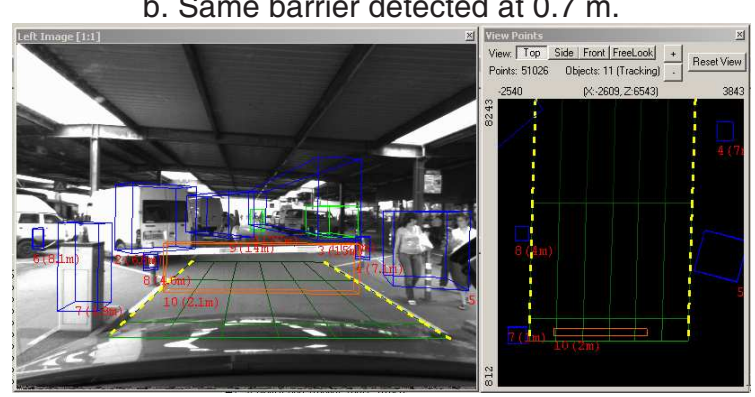

c. Detected barrier at $2.1 \mathrm{~m}$.

Figure 13. Detection results in different scenarios.
For barriers with no artifacts (few vertical edges / no visible texture) the maximum limit is reduced up to $6 \mathrm{~m}$ for (fig. 13.c). In the last case, the accuracy of the detected depth is lower due to the fact that the $3 \mathrm{D}$ information in the barrier's boom region is ambiguous.

Regarding the detection error rates, the true positive rate (TPR) was evaluated for a set of 8 different barrier scenarios, in a depth range from 0.7 .. $12 \mathrm{~m}$ (Table 2). Two cases were considered:

1. with applying the optional step (3.2 - figure 8): $\mathrm{TPR}_{1}$

2. without applying the optional step (3.2 -figure 8): $\mathrm{TPR}_{2}$

As expected the TPR decreases with the depth, the most reliable detection being encountered in the close range $(0.7 \ldots 4 \mathrm{~m})$. The average true positive rate was slightly better for the second case $\left(\mathrm{TPR}_{2}\right)$.

Table 2. True positive rates

\begin{tabular}{|l|c|c|}
\hline Depth Range & TPR $_{\mathbf{1}}[\%]$ & TPR $_{\mathbf{2}}[\mathbf{\%}]$ \\
\hline $\mathbf{0 . 7}$... 3.99 $\mathbf{~ m}$ & 99.58 & 99.79 \\
\hline $\mathbf{4 . 0} \ldots \mathbf{7 . 9 9} \mathbf{~ m}$ & 74.52 & 78.39 \\
\hline $\mathbf{8 . 0}$... 12.0 $\mathbf{~ m}$ & 67.23 & 70.59 \\
\hline Average & 86.71 & 88.59 \\
\hline
\end{tabular}

Regarding the false positive detection rate (FPR), the same 2 cases were considered for 3 different test scenarios (no barriers) with a total number of 670 images (Table 3 ). The false positive rate was slightly better for the first case $\left(\mathrm{FPR}_{1}\right)$.

Table 3. False positive rates

\begin{tabular}{|c|c|}
\hline FPR $_{\mathbf{1}}[\%]$ & $\mathbf{F P R}_{\mathbf{2}}[\%]$ \\
\hline 1.79 & 2.54 \\
\hline
\end{tabular}

Processing time depends on the number of horizontal edges detected in the 2D-ROI. In a scenario with no horizontal structures in the ROI the processing time is bellow $1 \mathrm{~ms}$ on an Intel E6750 processor. As the number of horizontal features increases, the processing time can increase up to $3 . .4 \mathrm{~ms}$. In a normal scenario with a true positive (detected barrier) the average processing time is about $2 \mathrm{~ms}$.

\section{Conclusions}

In this paper a barrier detection method was presented. The proposed method is able to detect horizontal structures (as barriers) which cannot be detected through the classical way as objects (stereo reconstruction and $3 \mathrm{D}$ points grouping). Therefore the barrier detection topic was not approached yet in the literature. 
The proposed method uses a hybrid approach: first detects the barriers in 2D and then infers the associated 3D information from dense stereo vision. The implementation of the method has real capabilities: the average processing overload of the method is around 2 $\mathrm{ms}$, depending on the scene complexity (number of horizontal edges), size of the 2D search space, number of horizontal lines detected through the Hough transform etc.

Many tests were performed to optimize the algorithms and to tune the parameters in order to minimize the false negatives and the false positives occurrences. The reported barriers are represented as common 3D objects bounded by a cuboid. Due to processing time constraints the orientation of the box was not computed.

The proposed method can be improved in order to extend the detection range and the robustness of the detection by:

- Using a drivable tunnel derived from the car dynamics and 3D elevations map [8] as search region in the $3 \mathrm{D}$ validation step in cases when the current lane information is not available.

- Performing the 2D detection and 3D validation on multi resolution images to increase the detection range (in the performed experiments images acquired by 1.3 MP cameras were down-sampled in order to meet the stereo-engine requirements [8]).

- Compensating the lack of 3D points in the case of barriers with no vertical features or texture by performing a software contour based correlation/reconstruction [16].

- Performing a 3D analysis of the barrier points in order to report an oriented box in the output objects list.

\section{References}

[1] The "PreVENT" Project, www.prevent-ip.org.

[2] The "Invent" Project, www.invent-online.de.

[3] Wikipedia - The Free Encyclopedia, http://en.wikipedia.org

[4] Toyota Motor Corporation, http://www.toyota.co.jp.

[5] BMW Corporation, http://www.bmw.com.

[6] A. Talukder, L. Matthies, Real-time detection of moving objects from moving vehicles using dense stereo and optical flow, Proceedings. of Intelligent Robots and Systems, IROS 2004), 28 Sept.-2 Oct. 2004, vol.4, pp. 3718- 3725.

[7] H. Badino, R. Mester, T. Vaudrey, U. Franke, Stereobased Free Space Computation in Complex Traffic Scenarios, IEEE Southwest Symposium Image Analysis and Interpretation, 2008. SSIAI 2008, 24-26 March 2008, Santa Fe, NM, pp. 189-192.

[8] S. Nedevschi, R. Danescu, T. Marita, F. Oniga, C. Pocol, S. Sobol, C. Tomiuc, C. Vancea, M. M. Meinecke, T. Graf, T. B. To, M. A. Obojski, A Sensor for Urban Driving Assistance Systems Based on Dense Stereovision, in Proc. Intelligent Vehicles (IV'07), Istambul, Turkey, 13-15 June 2007, pp. 278-286.

[9] S. Nedevschi, R. Danescu, D. Frentiu, T. Marita, F. Oniga, C. Pocol, S. Sobol, T. Graf, R. Schmidt, "Driving Environment Perception Using Stereovision," in Proc. IEEE Intelligent Vehicles Symposium (IV'05), Las Vegas, USA, June 2005, pp. 331-336.

[10] C. Pocol, S. Nedevschi, Marc-M. Meinecke, "Obstacle Detection Based on Dense Stereovision for Urban ACC Systems," in Proc. Workshop on Intelligent Transportation (WIT'08), Hamburg, Germany, 18-19 March 2008.

[11] R. Danescu, S. Nedevschi, M.M. Meinecke, T.B. To, "Lane Geometry Estimation in Urban Environments Using a Stereovision System", Proceedings of the IEEE Intelligent Transportation Systems Conference (ITSC 2007), Seattle, USA, 2007.

[12] R. C. Gonzalez , R. E. Woods, Digital Image Processing, Second Edition, Prentice-Hall, 2001.

[13] G.X. Ritter, J.N. Wilson, Handbook of computer vision algorithms in image algebra, 2-nd ed., CRC Press2001.

[14] S. Theodoridis, K. Koutroumbas, Pattern Recognition, 2-nd Edition, Academic Press, 2003.

[15] R. Danescu, S. Nedevschi, M.M. Meinecke, T. Graf, Stereovision Based Vehicle Tracking in Urban Traffic Environments, Proceedings of the IEEE Intelligent Transportation Systems Conference (ITSC 2007), Seattle, USA, 2007.

[16] S. Nedevschi, F. Oniga, R. Danescu, T. Graf, R. Schmidt, Increased Accuracy Stereo Approach for 3D Lane Detection, Proceedings of IEEE Intelligent Vehicles Symposium, (IV2006), June 13-15, 2006, Tokyo, Japan, pp. $42-49$. 